Article

\title{
Rebound Effect from Income Savings Due to an Energy Efficiency Improvement by Households: An Input-Output Approach
}

\author{
Taoyuan Wei ${ }^{1,2, *(1)}$ and Xue Wang ${ }^{1}$ \\ 1 School of Economics, Shandong University of Technology, Zibo 255049, China; wangxueok131@163.com \\ 2 CICERO Center for International Climate Research, 0318 Oslo, Norway \\ * Correspondence: taoyuan.wei@cicero.uio.no
}

Received: 14 July 2020; Accepted: 2 August 2020; Published: 5 August 2020

check for updates

\begin{abstract}
In previous studies on the indirect rebound effect due to income saved from energy efficiency improvement by households in an input-output model, household income is typically assumed constant before and after an efficiency improvement of their energy use. The assumption is implausible if we observe that households may not re-spend the saved income, which potentially reduces their consumption and national income including households, at least in the short term, in a country like China, where they have high saving propensity. In addition, income of other economic agents than households may also be affected. In this article, we provide an improved input-output model to clarify the issue and then use the 2017 input-output data of China to illustrate the potential indirect rebound effect due to household income saved from an energy efficiency improvement. Our results show that if the income impact in the short term is properly considered, then the indirect rebound effect due to the income savings could be trivial at the national level while still considerable at the household level. Hence, the indirect rebound effect at the household level alone is likely misleading for national policy makers by providing an overestimated value of the short-term indirect rebound effect.
\end{abstract}

Keywords: input-output; income savings; indirect rebound effect; households; China

\section{Introduction}

Worldwide, households consume $21 \%$ of total energy for final use, and in China, the share was $17 \%$ in 2017 [1]. In recent years, China has taken measures to encourage more domestic consumption, which may lead to more energy use by households. While total emissions can be reduced if households consume more and save less in China, due to lower energy-intensity of household consumption than that of investments [2], the energy and emissions from the household sector could increase in the future and play a more important role for energy savings and climate mitigation. Hence, it is crucial for households to take actions to reduce energy use while achieving the same level of well-being. One of the measures commonly recommended and widely accepted as cost-effective is efficiency improvement in energy use. However, it is a controversial issue, regarding the energy savings from energy efficiency improvements by households when considering the behavior changes of households and other relevant economic agents.

An energy efficiency improvement can directly lead to less energy used by households to obtain the same level of energy services. Consequently, households will spend less on energy use even if energy price is constant (indeed, the reduced energy demand can potentially pull down the energy price and result in an additional reduction in spending). The reduced cost on energy use may encourage households to use more energy to obtain a higher level of energy services and/or re-spend on other products/services if their income is not affected by the energy efficiency improvement. 
The phenomenon of more energy use associated with an energy efficiency improvement is called the "rebound effect" in the literature [3,4]. Following Khazzoom [5], Greening et al. [6] and later literature, the increase in energy services due to lower cost of the services alone, assuming constant energy price at the micro-level, is classified as a direct rebound effect of an energy efficiency improvement. For example, a person may drive more if fuel cost per kilometer is lowered by an efficiency improvement of the fuel. The re-spending on additional goods of the saved income by households is classified as indirect rebound effect. The energy efficiency improvement may also induce changes in market prices of energy and other goods, disturbing the whole economic system, which is classified as economy-wide rebound effect.

One of the typical methods to study the direct and indirect rebound effects in the household sector is combining a re-spending mechanism with input-output modeling and simulation, where market prices are constant and production technologies are fixed, excluding the economy-wide rebound effect $[7,8]$. We can observe that there are other approaches to examining the rebound effects in the literature including econometric modeling (e.g., [9-12]) and computable general equilibrium modeling $[13,14]$. Each approach has its limitations and constraints that might be addressed by other approaches.

In the studies on the rebound effect based on the input-output modeling and simulation [15-22], a typical assumption is that the income of households stays constant before and after the introduction of an energy efficiency improvement, although various methods are used to describe the re-spending mechanism for identification of the direct rebound effect $[7,8,21]$. The assumption is likely valid if households still expect to receive the same income as before and re-spend on additional consumption, particularly in developed countries with low saving propensity.

In a developing country with high saving propensity like China, however, the re-spending might unlikely occur since most of the households tend to save as much as they can. In principle, the additional savings enter the financial sectors and potentially can be used for investment to induce more activities in the real economy, but it is unlikely in the short term, i.e., within a year. Hence, the energy efficiency improvement may reduce total demand, resulting in less economic activities that generate income of economic agents including households, at least in the short term. In this sense, the assumption of constant household income is questionable in the input-output framework and may provide biased information on the indirect rebound effect of an energy efficiency improvement, particularly because almost all the households implement a common efficiency improvement in energy use (e.g., a more efficient stove or car is adopted by most of the households).

If households in China improve their energy efficiency by $20 \%$, for example, the energy use of the households is expected to reduce by $20 \%$ assuming no rebound effect. Since $17 \%$ of total energy at the national level is used by households, total national energy demand would decline by $3.4 \%$ correspondingly, which implies a reduction in income of the energy production sectors since the energy supply has to be reduced by the same $3.4 \%$ in the input-output framework. At least some part of the income reduction goes to the household sector, which conflicts with the assumption of constant income in previous studies. Hypothetically, if all the income reduction goes to the household sector, then the income corresponding to the reduced energy use disappears, meaning no indirect rebound effect from income savings due to the energy efficiency improvement.

This article contributes to the literature on the rebound effect by the input-output modeling by considering the potential disturbance on income of relevant economic agents of an energy efficiency improvement by households. Although still assuming constant market prices and fixed production technologies, the income disturbance caused by the energy efficiency improvement can still result in the indirect rebound effect, which may markedly differ from the case of constant income of households and other economic agents. The potential range of the indirect rebound effect from the income disturbance in an input-output framework is illustrated by the case of households in China based on the latest input-output data of China in 2017 [23]. 


\section{Methods}

\subsection{The Input-Output Model Considering Income Allocation}

The input-output model, initially developed by Leontief [24], considers the interactions between production sectors in an economy by assuming linear fixed coefficients of sectoral inputs to a production sector. The market prices are implicitly assumed constant and substitutions between any two of intermediate and primary inputs are not allowed in the modelling world. The method is based on data of input-output tables, which are widely applied to assess the sectoral interactions and impacts of economic activities [25].

In this study, we assume an open economy produces a set of products $N=\{1,2, \ldots, n\}$, which is used for satisfying intermediate production and final demand. In an input-output model, the production of all the products can be expressed in the matrix form,

$$
x=(I-A)^{-1}\left(f^{h}+f^{o}+e\right)
$$

where $x=\left(x_{1}, x_{2}, \ldots, x_{n}\right)^{\prime}$ is the n-dimensional column vector of output, $f^{h}=\left(\begin{array}{ccc}f_{1}^{h} f_{2}^{h} & \ldots & f_{n}^{h}\end{array}\right)^{\prime}$ is the n-dimensional column vector of household consumption of domestically produced goods, $f^{o}=\left(\begin{array}{lll}f_{1}^{o} f_{2}^{o} & \ldots & f_{n}^{o}\end{array}\right)^{\prime}$ is the n-dimensional column vector of final demand for domestically produced goods of economic agents other than households including government and investors, and $e=$

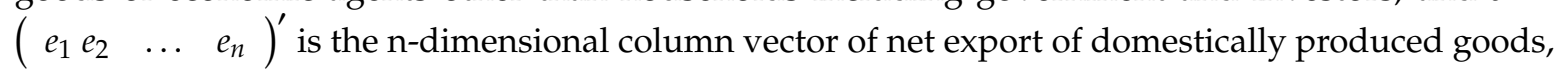
$\boldsymbol{I}$ is an n-dimensional identity matrix, and $(\boldsymbol{I}-\boldsymbol{A})^{-1}$ is the well-known Leontief inverse matrix, where $\boldsymbol{A}$ is the n-dimensional matrix of intermediate consumption coefficients during the production activities with each element $a_{i j}^{d}$ representing the input of Product $i$ (either domestic produced or imported) to produce one unit of Product $j$,

$$
A=\left[\begin{array}{cccc}
a_{11} & a_{12} & \cdots & a_{1 n} \\
a_{21} & a_{22} & \cdots & a_{2 n} \\
& \cdots & \cdots & \cdots \\
a_{n 1} & a_{n 2} & \cdots & a_{n n}
\end{array}\right]
$$

We can decompose the domestic final demand into two terms,

$$
f^{h}=s^{h} \cdot y^{h}, F^{o}=s^{o} \cdot y^{o}
$$

where $y$ is income of corresponding final demanders and $s$ is the column vector of shares of goods consumed by the corresponding final demanders. A change in household income $\left(y^{h}\right)$ due to an energy efficiency improvement then leads to a change in final consumption and associated energy use, which is the basis to estimate the indirect rebound effect.

The model above is typically adopted in previous studies on the indirect rebound effect without involving income flows, although income of households alone is assumed constant. In this study, we consider an improved input-output model that also includes a description of income flows as follows.

The income of final demanders comes from the value added $(v)$ generated from production activities, which is initially allocated to owners of the primary inputs as compensation of employees, and returns to capital assets, and production taxes to the government who provides public services,

$$
v=A^{v} x
$$

where $(v)$ is an n-dimensional matrix of primary factor income and $A^{v}$ is an n-dimensional diagonal matrix, 


$$
A^{v}=\left[\begin{array}{cccc}
a_{11}^{v} & 0 & \cdots & 0 \\
0 & a_{22}^{v} & \cdots & 0 \\
& \cdots & \cdots & \cdots \\
0 & 0 & \cdots & a_{n n}^{v}
\end{array}\right]
$$

And $a_{\mathrm{jj}}^{\mathrm{v}}=1-\sum_{\mathrm{i}=1}^{\mathrm{n}} \mathrm{a}_{\mathrm{ij}}$ for $\mathrm{j} \in\{1,2, \ldots, \mathrm{n}\}$.

We can observe that on the one hand, output of a product is the sum of intermediate input and value added. On the other hand, it is the sum of intermediate input and final demand. Hence, the value added is equal to total final demand, i.e.,

$$
i^{r} v=i^{r} f^{h}+i^{r} f+i^{r} e
$$

where $\mathrm{i}^{\mathrm{r}}=\left(\begin{array}{llll}1 & 1 & \ldots & 1\end{array}\right)$ is an $\mathrm{n}$-dimensional unit row vector. Behind the equation is the re-allocation of value added as income among final demanders to generate domestic final demand for consumption and investments as well as the net foreign demand.

\subsection{Income Impact of an Energy Efficiency Improvement by Households}

Assuming an energy efficiency improvement, households then reduce energy use by an amount of $\Delta f^{h}$ with non-zero element for the energy products alone. By Equation (1), we can calculate its impact on sectoral output as $\Delta x$, which is then used in Equation (3) to calculate the impact on sectoral income,

$$
\Delta v=A^{v} \Delta x=A^{v}(I-A)^{-1} \Delta f^{h}
$$

Which sums up across sectors is equal to the reduced spending on energy use by the households in the input-output modelling world.

The income reduction from production activities is allocated to primary factors including wages to labours provided by households, profits to capital owners, and taxes to the government. After income re-allocation in the form of transfers among various economic agents, the income reduction is finally received by the economic agents including households, governments, companies, and foreigners, who further decide how much for consumption and savings, which then affect energy used during the adjustment of the consumption and investments. In a typical input-output table, the re-allocation of income among economic agents is missing.

Previous input-output studies on indirect rebound effect argue that households could re-spend their income savings from reduced energy use on any consumption goods [15-22]. This implicitly assumes that the income of households is constant without any impact of the energy efficiency improvement measures. The assumption might be true for some households but unlikely for all the households if considering the response of producers to the reduced energy demand in the market. Since income are likely reduced in the production, the income of all the economic agents including households could not hold constant even within a year. Hence, at the aggregate level, the income of economic agents including households would not be the same as the level before the energy efficiency improvement.

\subsection{Indirect Rebound Effect from Income Re-Allocation}

If we assume all the expected income reduction are received by the households, then income saved by households would disappear and no indirect rebound effect can be generated from the income savings. If we assume the expected income reduction are also received by economic agents other than the households, then households may keep part of the income savings and generate certain indirect rebound effect.

To provide a complete story about the indirect rebound effect from the income savings of an energy efficiency improvement by households, we consider how the income reduction is received by various economic agents including households. 
First, we figure out how much expected income savings due to an energy efficiency improvement. If no rebound effect, then a $20 \%$ improvement of energy efficiency results in a $20 \%$ reduction in energy use and associated spending, i.e., a 20\% income savings. However, this will not occur if we consider the direct rebound effect: the energy services provided by the energy use become cheaper and induce more demand for the energy use. If assuming the direct rebound effect is a\%, then we would expect the income savings of only $(1-a \%) \cdot 20 \%$.

Second, the $(1-a \%) \cdot 20 \%$ income savings could not be kept by households alone as we argued by the improved input-output model above. The corresponding $(1-a \%) \cdot 20 \%$ reduction in energy use leads to shrunk production activities and income generation, which is then received by economic agents including typically households, government, and investors. Hence, in the input-output model presented above, Equation (5) have to be modified to include the effect on income of other economic agents $\left(f^{o}\right)$,

$$
\Delta v=A^{v} \Delta x=A^{v}(I-A)^{-1}\left(\Delta f^{h}+\Delta f^{0}\right)
$$

If we assume no changes in net export $(e)$.

Finally, the economic agents would adapt to the reduced income by less consumption and investments and related energy use, resulting in indirect rebound effect. This implies that the indirect rebound effect can be generated by not only the households, but also other economic agents.

Following previous input-output studies on indirect rebound effect $[7,8,26]$, the indirect rebound effect can be calculated by the general definition of rebound effect $(R)$.

$$
R=\frac{P E S-A E S}{P E S}
$$

where PES refers to potential (or expected) energy savings from an energy efficiency improvement assuming no rebound effect and AES refers to the "actual" energy savings. In our case of an $20 \%$ improvement of energy efficiency, the potential energy savings (PES) is $20 \%$ of the energy use before the introduction of the efficiency improvement. The "actual" energy savings $(A E S)$ is the difference between the energy use in the input-output model before and after the income savings is induced by the efficiency improvement. We assume that the direct rebound effect is given exogenously in this study.

In this article, we consider three scenarios of the income re-allocation among economic agents.

The Worst Scenario for households. Income of households is reduced at the same level as their savings from the reduction in energy use. This way, there is no indirect rebound from income savings, although this is unlikely the actual case.

The Best Scenario for households with "proportional re-spending" as stated in Lu and Wang [22]. This scenario adopts the same assumption of constant household income as previous studies on indirect rebound effect, i.e., the reduction of income does not occur. This implicitly assumes that the domestic production activities are not affected, which can be true if either the saved energy is exported to receive the same payment from abroad, or the imported energy is reduced at the same level as the saved energy at the same value. Indirect rebound occurs and adds up to the direct rebound.

The Medium Scenario for households. The reduced income is proportionally allocated to households, government, and investors while the foreign agents receive the same income as before. As a result, households still receive part of the income savings, which is equal to the sum of the reduced income of the government and investors. How energy use evolves with the income re-allocation depends on the difference of energy intensity between households and the other two economic agents of government and investors.

\section{Data}

We illustrate the indirect rebound effect from income savings due to an energy efficiency improvement by the case of electricity used by households in China. Our illustration is based on the input-output table of China 2017 [23], where the economy in China is divided into 149 production 
sectors including six energy sectors of raw coal, crude oil and natural gas, refined oil products, coal products, fuel gas and electricity. For simplicity without loss of generality, we assume a $20 \%$ efficiency improvement of electricity used by the households in China. We can observe that the results on the indirect rebound effect of this study will not change if we assume any reasonable value other than $20 \%$ of energy efficiency improvement since the economic activities are described in linear functions.

To estimate the indirect rebound effect from income saved by households, we must know the direct rebound effect of the energy efficiency improved by the households. In this study, we refer to the previous studies about the range of the direct rebound effect. Ouyang et al. [27] adopted a high direct rebound effect of 30\% and 50\%. Wen, Ye, Yang and Li [19] conclude with a much smaller range of direct rebound effect of 14-20\% based on provincial data in China. Li et al. [28] found the direct rebound effect of around $66 \%$ for urban households. Focusing on electricity used by households in China, the direct rebound effect for all households is found to be $72 \%$ [29] and 85\% [11], while Han et al. [30] estimated a range from $37 \%$ to $50 \%$ of direct rebound effect of urban households. Hence, we consider that the direct rebound effect ranges from $15 \%$ to $85 \%$ for each scenario in this study.

\section{Results and Discussion}

Figure 1 shows the indirect rebound effects due to an efficiency improvement of electricity used by households in China for various direct rebound effect values.

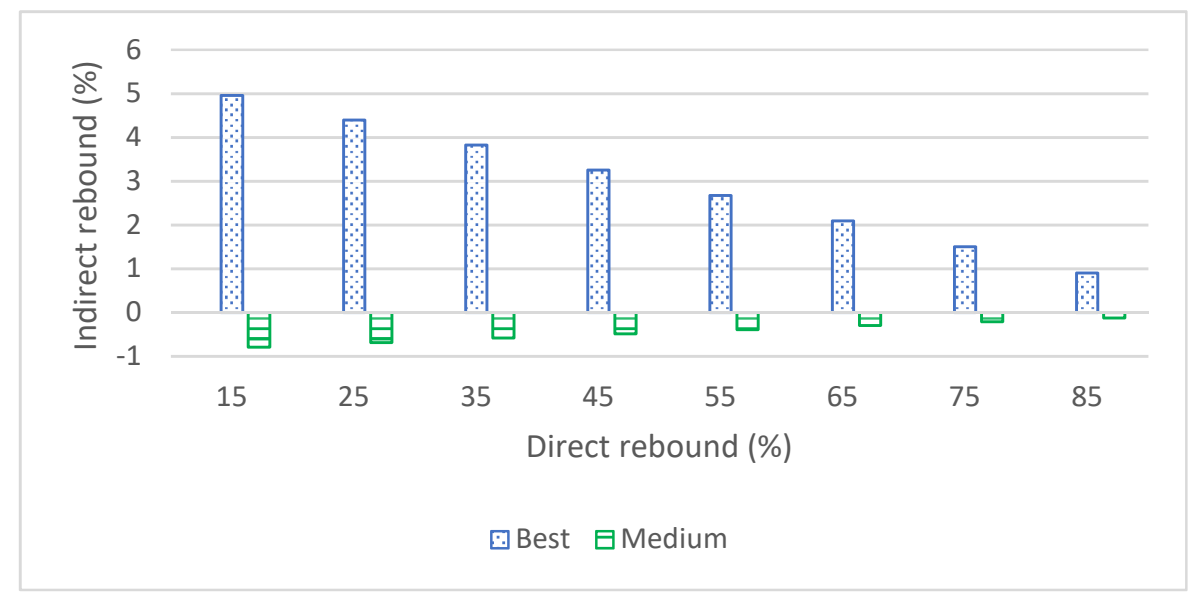

Figure 1. The indirect rebound effect at the national level with various assumed direct rebound effect of an efficiency improvement of electricity used by households in China. The calculation is based on an input-output model using the 2017 data.

In the Worst Scenario for households, the reduced electricity use induced by the $20 \%$ improvement in the electricity efficiency leads to income reduction at the same level as the income savings, based on the input-output modelling assumptions. Hence, this scenario corresponds to the case of no expected income savings and no indirect rebound effect no matter how the direct rebound effect is. A special example of this scenario could be that the households themselves generate part of the electricity for their own use at a relatively high cost, particularly in some developing areas. When they improve by $20 \%$ efficiency in electricity use, they would directly reduce their own electricity use and production to save cost. Hence, the cost/income savings induced from the efficiency improvement disappears simultaneously without any indirect rebound effect, although certain direct rebound effect might still be there. This conclusion likely holds for other economic agents who produce energy for their own consumption.

Opposite to the Worst Scenario, previous studies assume scenarios like the Best Scenario in this study where households in China were assumed to keep the same income level as before the efficiency improvement in electricity use. As shown in Figure 1, the indirect rebound effect in the Best Scenario ranges from $1 \%$ to $5 \%$ depending on the assumed direct rebound effect in the case of households 
in China. A higher direct rebound effect corresponds to a lower indirect rebound effect. Since the indirect rebound effect is relatively smaller than the direct rebound effect, the total of direct and indirect rebound effects is dominated by the direct rebound effect, although the indirect rebound effect is relatively stronger in the cases of lower direct rebound effect.

In the Best Scenario for households, the other domestic economic agents are implicitly assumed to keep the same level of their income as before, while the possible income reduction goes to foreigners, who are out of the consideration of households and relevant stakeholders in China. Hence, the indirect rebound effect might be overestimated for China since the effect on foreign countries is ignored.

So far, the indirect rebound effect is the same for households and for China as a nation, since no income changes are assumed for economic agents other than households in China. In the Medium Scenario, the income of government and investors is reduced, and the indirect rebound effect of households differs from that of China as a nation.

In the Medium scenario, the indirect rebound effect at the national level is lowered considerably to become negative and less important with higher direct rebound effect (Figure 1). This is because the reallocation of income leads to energy savings at the national level rather than more energy use, implying that the indirect rebound effect would mitigate the positive direct rebound effect. However, with a higher direct rebound effect, the level of income reduction is lowered, limiting the induced indirect rebound effect. The negative indirect rebound effect is due to the energy intensity of government and investors being greater than that of households, consistent with the findings of Lin, Qi, Liang, Feng, Wiedmann, Liao, Yang, Li, Mi and Yang [2].

If we focus only on households, the indirect rebound effect of households alone is positive rather than negative, although the values become smaller with a higher direct rebound effect (Figure 2). If we only report the indirect rebound effect at the households' level, we overestimate the indirect rebound effect considerably, e.g., a $2 \%$ rather than $-0.5 \%$ indirect rebound effect in the case of $45 \%$ direct rebound effect.

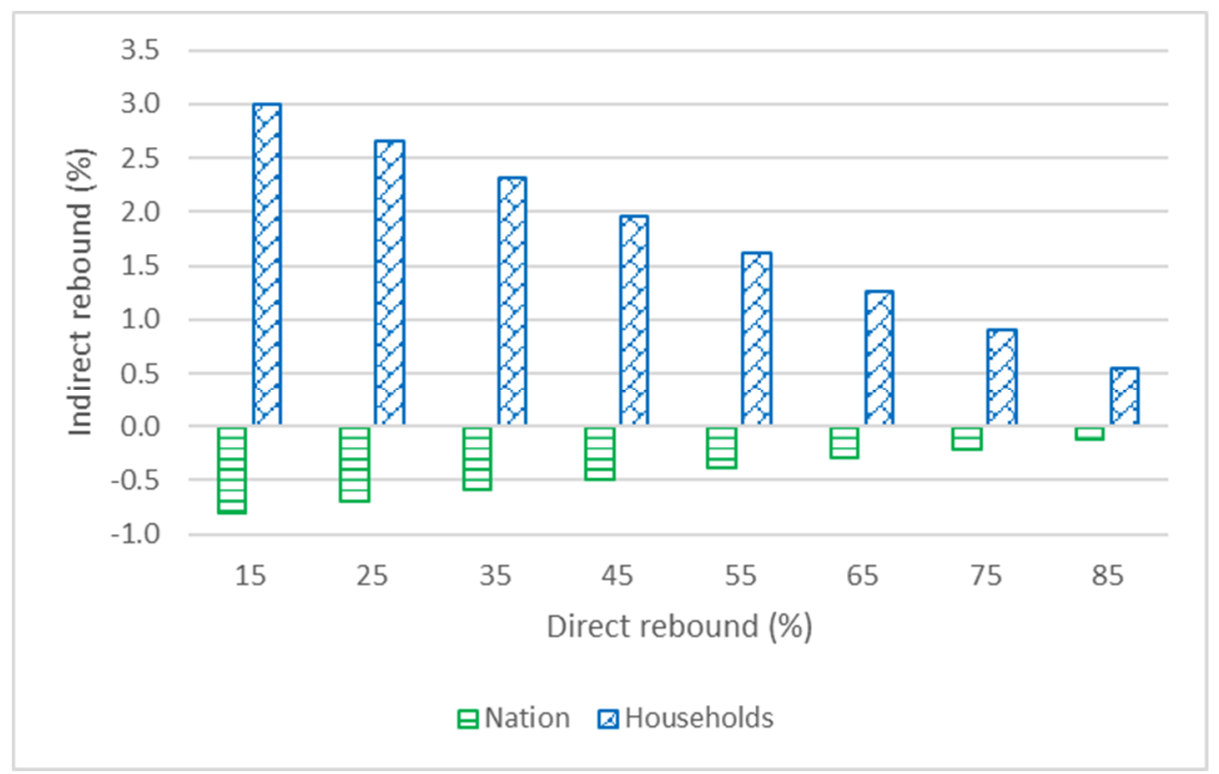

Figure 2. The indirect rebound effect at the national level and at the households' level in the Medium Scenario with various assumed direct rebound effect of an efficiency improvement of electricity used by households in China. The calculation is based on an input-output model using the 2017 data.

It seems that if we include more economic agents in the analysis, the indirect rebound effect tends to become less important since the income savings of households corresponds to an income reduction of other agents in the economy. Generally, the energy intensity does not differ considerably across 
households and other economic agents, meaning there is a trivial indirect rebound effect from income savings in an input-output modelling world.

\section{Comparison with Results in Lu and Wang}

Regarding previous studies, Lu and Wang [22] is the most relevant one to our study and is representative since they estimated the rebound effect for the electricity used by urban households in China based on the 2007 input-output data. According to their estimates, the indirect rebound effect is in a range of $18-20 \%$ in the case of direct rebound effect of $72-74 \%$. In our case, however, we obtain a much smaller indirect rebound effect of only about $1.5 \%$ with a $75 \%$ direct rebound effect. The difference could be caused for several reasons.

First, the data used by Lu and Wang [22] are 10 years older than ours. The economic structure and scale have changed considerably during those 10 years in China. For example, the share of coal-fuelled electricity generation in total has declined from $81 \%$ in 2007 [31] to $68 \%$ In 2017 [32]. Another relevant study is Wen, Ye, Yang and Li [19], who estimated the direct rebound effect of $17 \%$ and the indirect rebound effect of $57.5 \%$ on average based on provincial data of China. The indirect rebound at the provincial level varies in a large range of $20-165 \%$, while the direct rebound is in a much smaller range of $14-20 \%$. Their results seem to be opposite of that of Lu and Wang [22]. This may also indicate the considerable impact of data sources on the estimated results of the rebound effects.

Second, and probably the most important, we consider the indirect rebound effect on electricity use alone, while Lu and Wang [22] seem to consider all the energy use induced by an efficiency improvement of electricity used by households. In China, most of the electricity is generated from coal. If the coal used to generate electricity is accounted for in the energy use, then the "actual" energy savings $(A E S)$ calculated from the input-output model could be much larger, leading to much greater indirect rebound effect, since the expected energy savings (PES) refer to electricity use only, rather than all energy used by households. In our opinion, while the efficiency improvement of electricity use could also induce changes in the use of other non-electricity energy goods, we limit our attention to electricity use alone to avoid a possible double-accounting issue in this study.

Third, we consider the electricity used by all households rather than the urban part alone. The urban households use much more electricity than rural households. When we include the rural households, the re-spending structure of households differ from that of the urban households alone.

\section{Conclusions}

In this study, we critically discussed the possible indirect rebound effect from income savings due to an efficiency improvement of energy use in households. We consider three alternative scenarios for the re-allocation among economic agents of the income savings due to an efficiency improvement of energy used by households. Our results show that the indirect rebound effect of households could be zero, positive, or negative, depending on different assumptions of the income re-allocation. We also find that the indirect rebound effect at the national level can differ from that at the household level, indicating the indirect rebound effect estimated from partial economic models may be misleading for national policy makers for a developing country like China, where households have relatively high saving propensity.

Our results highlight the importance of income re-allocation among economic agents for estimating the rebound effect, which should be considered carefully by energy efficiency policy makers, although this study assumes that the energy production is reduced at the same level as the reduced energy use by households due to an energy efficiency improvement. This assumption is plausible in the short term but unlikely in the long term when the adjustment of economic activities becomes more flexible. In this sense, our estimation of the indirect rebound effect may be lower than that the potential long-term indirect rebound effect.

Moreover, our study highlights that energy production activities can be reduced by an energy efficiency improvement, at least in the short term. This could be a barrier for policies to improve 
energy efficiency since reduced energy production may lead to unemployment and lower capital utility rates if market responses are not in place in the short term. Hence, as a supplement to measures for energy efficiency policy without income losses even in short term, it is recommended to encourage more investments and employment for non-energy production activities to reduce energy use. If the energy efficiency policy seeks emission abatement rather than energy reduction, then renewable energy activities can be encouraged, too.

In the illustrated case of households in China, we used several assumptions to simplify the analysis, possibly resulting in an underestimated indirect rebound effect. One of the assumptions is that in the calculation, we consider only electricity use affected by the efficiency improvement of electricity use by households, although the use of other non-electricity energy goods may also be affected. By doing so, we also avoid possible double accounting for induced energy use in the energy transformation process. For example, the induced increase in electricity use likely needs more coal use in China, as coal-fuelled electricity generation accounts for nearly $60 \%$ of total electricity generation in 2017. It would be implausible to consider the increase of both electricity and coal use simultaneously in the calculation. Another study on the role of energy transformation in the estimated indirect rebound effect would be useful.

Like other studies based on input-output modelling, this study assumes constant prices and linear production technologies, which exclude market responses to the energy efficiency improvement. Hence, the results are more plausible to be interpreted as a short-term rather than long-term indirect rebound effect. In the long term, the income might not be reduced at all if the resources used to produce the saved energy are re-allocated to produce other products besides re-allocation within the same production sector.

Author Contributions: Conceptualization, methodology, formal analysis, writing—original draft preparation, and writing - review and editing, T.W.; data curation and writing—review and editing, X.W. All authors have read and agreed to the published version of the manuscript.

Funding: This research received no external funding.

Conflicts of Interest: The authors declare no conflict of interest.

\section{References}

1. IEA. World Transition Indicators 2019 Edition. Available online: https://iea.blob.core.windows.net/assets/ 73f40d94-d523-4d1b-9084-7029a33ed8fa/TransitionIndicators_Documentation.pdf (accessed on 20 April 2020).

2. Lin, C.; Qi, J.; Liang, S.; Feng, C.; Wiedmann, T.O.; Liao, Y.; Yang, X.; Li, Y.; Mi, Z.; Yang, Z. Saving less in China facilitates global $\mathrm{CO}_{2}$ mitigation. Nat. Commun. 2020, 11, 1358. [CrossRef]

3. Saunders, H.D. The Khazzoom-Brookes postulate and neoclassical growth. Energy J. 1992, 13, 131-149. [CrossRef]

4. Saunders, H.D. A view from the macro side: Rebound, backfire, and Khazzoom-Brookes. Energy Policy 2000, 28, 439-449. [CrossRef]

5. Khazzoom, J.D. Economic implications of mandated efficiency in standards for household appliances. Energy J. 1980, 1, 21-40.

6. Greening, L.; Greene, D.L.; Difiglio, C. Energy Efficiency and Consumption—The Rebound Effect-A Survey. Energy Policy 2000, 28, 389-401. [CrossRef]

7. Thomas, B.A.; Azevedo, I.L. Estimating direct and indirect rebound effects for U.S. households with input-output analysis Part 1: Theoretical framework. Ecol. Econ. 2013, 86, 199-210. [CrossRef]

8. Freire-González, J. Methods to empirically estimate direct and indirect rebound effect of energy-saving technological changes in households. Ecol. Model. 2011, 223, 32-40. [CrossRef]

9. Orea, L.; Llorca, M.; Filippini, M. A new approach to measuring the rebound effect associated to energy efficiency improvements: An application to the US residential energy demand. Energy Econ. 2015, 49, 599-609. [CrossRef]

10. Adetutu, M.O.; Glass, A.J;; Weyman-Jones, T.G. Economy-wide estimates of rebound effects: Evidence from panel data. Energy J. 2016, 37, 251-269. [CrossRef] 
11. Xin-gang, Z.; Pei-ling, L. Is the energy efficiency improvement conducive to the saving of residential electricity consumption in China? J. Clean. Prod. 2020, 249, 119339. [CrossRef]

12. Shao, S.; Guo, L.; Yu, M.; Yang, L.; Guan, D. Does the rebound effect matter in energy import-dependent mega-cities? Evidence from Shanghai (China). Appl. Energy 2019, 241, 212-228. [CrossRef]

13. Wei, T.; Liu, Y. Estimation of global rebound effect caused by energy efficiency improvement. Energy Econ. 2017, 66, 27-34. [CrossRef]

14. Madlener, R.; Turner, K. After 35 years of rebound research in economics: Where do we stand? In Rethinking Climate and Energy Policies; Springer: New York, NY, USA, 2016; pp. 17-36.

15. Freire-González, J. Evidence of direct and indirect rebound effect in households in EU-27 countries. Energy Policy 2017, 102, 270-276. [CrossRef]

16. Freire-González, J.; Font Vivanco, D.; Puig-Ventosa, I. Economic structure and energy savings from energy efficiency in households. Ecol. Econ. 2017, 131, 12-20. [CrossRef]

17. Thomas, B.A.; Azevedo, I.L. Estimating direct and indirect rebound effects for U.S. households with input-output analysis. Part 2: Simulation. Ecol. Econ. 2013, 86, 188-198. [CrossRef]

18. Wang, Z.; Han, B.; Lu, M. Measurement of energy rebound effect in households: Evidence from residential electricity consumption in Beijing, China. Renew. Sustain. Energy Rev. 2016, 58, 852-861. [CrossRef]

19. Wen, F.; Ye, Z.; Yang, H.; Li, K. Exploring the rebound effect from the perspective of household: An analysis of China's provincial level. Energy Econ. 2018, 75, 345-356. [CrossRef]

20. Lekve Bjelle, E.; Steen-Olsen, K.; Wood, R. Climate change mitigation potential of Norwegian households and the rebound effect. J. Clean. Prod. 2018, 172, 208-217. [CrossRef]

21. Wang, L.; Zha, D.; Wu, F. Re-examining hybrid methodologies for estimating residential rebound effects. Energy Effic. 2019, 12, 933-945. [CrossRef]

22. Lu, M.; Wang, Z. Rebound effects for residential electricity use in urban China: An aggregation analysis based E-I-O and scenario simulation. Ann. Oper. Res. 2017, 255, 525-546. [CrossRef]

23. NBSC. Input-output Tables of China 2017. Available online: http://data.stats.gov.cn/ifnormal.htm?u=/files/ $\mathrm{html} /$ quickSearch/trcc/trcc01.html\&h=740 (accessed on 20 April 2020).

24. Leontief, W.W. The Structure of American economy, 1919-1939: An Empirical Application of Equilibrium Analysis; Oxford University Press: New York, NY, USA, 1951.

25. Miller, R.E.; Blair, P.D. Input-Output Analysis: Foundations and Extensions; Cambridge University Press: New York, NY, USA, 2009.

26. Druckman, A.; Chitnis, M.; Sorrell, S.; Jackson, T. Missing carbon reductions? Exploring rebound and backfire effects in UK households. Energy Policy 2011, 39, 3572-3581. [CrossRef]

27. Ouyang, J.; Long, E.; Hokao, K. Rebound effect in Chinese household energy efficiency and solution for mitigating it. Energy 2010, 35, 5269-5276. [CrossRef]

28. Li, X.; Liu, J.; Liu, X. Direct rebound effect for urban household in China-An empirical study. Energy Effic. 2017, 10, 1495-1510. [CrossRef]

29. Zhang, Y.-J.; Peng, H.-R. Exploring the direct rebound effect of residential electricity consumption: An empirical study in China. Appl. Energy 2017, 196, 132-141. [CrossRef]

30. Han, Y.; Shi, J.; Yang, Y.; Wang, Y. Direct Rebound Effect for Electricity Consumption of Urban Residents in China Based on the Spatial Spillover Effect. Energies 2019, 12, 2069. [CrossRef]

31. IEA. World Energy Outlook 2009; International Energy Agency: Paris, France, 2009.

32. IEA. World Energy Outlook 2019; International Energy Agency: Paris, France, 2019.

(C) 2020 by the authors. Licensee MDPI, Basel, Switzerland. This article is an open access article distributed under the terms and conditions of the Creative Commons Attribution (CC BY) license (http://creativecommons.org/licenses/by/4.0/). 